\title{
Adapting a Multimedia Distance Learning Environment for Vision Impairments
}

\author{
Vidhya Balasubramanian and Nalini Venkatasubramanian \\ Department of Information and Computer Science \\ University of California, Irvine, Irvine California-92697-3425, USA \\ \{vbalasub,nalini\}@ics.uci.edu
}

\begin{abstract}
Distance learning applications provide valuable opportunities to users with disabilities. To enhance the accessibility of the distance-learning portal to vision impaired users, we utilize analytical studies on human vision to develop a visibility based adaptation algorithm. Using this adaptation the quality levels and layout of the portal is adapted to enhance the visibility, to users with various vision impairments.
\end{abstract}

\section{Introduction}

Distance learning is an application that can provide excellent opportunities for users with disabilities. However, the information provided to the user should be suitably adapted, so that it is maximally accessible to the users with different impairments. In this paper we discuss how we use different techniques to adapt the distance learning application to specifically suit users with different vision impairments. In this study we consider a variety of vision disorders like refractive error, glaucoma, cataract etc., where transcoding techniques are applied to change the quality of the image and text information. The quality in this context encompasses resolution, color contrast, brightness, and font size, and also the layout of the distance-learning portal. The adaptation techniques proposed cater to varying levels of visual perception that a user possesses. Specifically, we derive from models of human visual systems to determine suitable transcoding functions for effective layout of the interface.

Accessibility of multimedia information to vision impaired users has been studied from different angles and various solution have been proposed. One common solution is the use of assistive devices to enhance visibility of the information. Tools such as screen readers, audio HTML interfaces and auditory navigation alleviate some problems faced by users with impaired vision [10, $14,15]$. Manipulation of pointers to assist low vision users has been proposed in [2]. In this paper we concentrate on providing a generic solution to improving accessibility of the information sent to users with vision impairments using comprehensive studies on human vision. This paper specifically caters to the class of users with various degrees of vision impairments, (with at least some level of light perception) and proposes a middleware solution to enhancing visual accessibility.

\section{Visibility Based Adaptation}

An understanding of the human visual system is essential for effective adaptation of the distance-learning environment for different vision impairments. According to the different studies on the human visual system, $[13,1,11,12]$ effective visual perception is dependent on a number of factors. These factors are as follows:

Visual Acuity (V): It is the ability to resolve a spatial pattern separated by a visual angle $(\alpha)$ of 1 minute of arc. In the context of textual information, it is the ability to resolve contrast on a white background that subtends 5 minutes of arc. Using the common Snellen fraction, visual acuity is 20/20 for a normal user.

$$
\mathrm{V}=1 / a \text { where } \alpha=h / d * 3438 \text { minutes }
$$

where $h$ and $d$ are the height and viewing distance of the media information.

Contrast Sensitivity (S): It is a measure of the limit of visibility of low contrast patterns before they become indistinguishable from a uniform field. It is the function of the coarseness/fineness of image features.

Brightness (B): Brightness is the subjective evaluation or interpretation of the amount of light reaching the visual system. For simplicity it can be related to the luminance of the image, which is the product of light, reflected and light reaching the surface. It varies logarithmically with luminance.

Color Perception (C): For an arbitrary image..the three responses are $X, Y, Z$ (also called tristimulus values) for red, green and blue, can be calculated from the RGB color values as follows 
Table 1: Adaptation policies for different Vision Impairments

\begin{tabular}{|c|c|c|c|}
\hline Impairment & $\begin{array}{l}\text { Affected Part of } \\
\text { eye and effect }\end{array}$ & Factor affected & Transcoding required \\
\hline $\begin{array}{l}\text { Refractive errors (near } \\
\text { vision, far vision, } \\
\text { presbiopia) }\end{array}$ & $\begin{array}{l}\text { Lens which results in } \\
\text { poor focus and hence } \\
\text { blurred image }\end{array}$ & $\begin{array}{l}\text { Visual acuity (v) } \\
\operatorname{Norm}(\mathrm{v})=\mathrm{v}\end{array}$ & $\begin{array}{l}\text { For given acuity } v \text { and size of image or } \\
\text { text } y, \text { new size } Y=\text { size } / v \\
\text { New norm }(v)=y^{*} \text { / size }\end{array}$ \\
\hline $\begin{array}{l}\text { Cataract and Corneal } \\
\text { Pathology }\end{array}$ & $\begin{array}{l}\text { Affects lens resulting } \\
\text { in hazy, distorted } \\
\text { vision }\end{array}$ & $\begin{array}{l}\text { Visual acuity }(\mathrm{v}), \\
\text { contrast sensitivity } \\
\text { Norm }(\mathrm{v})=\mathrm{v} ; \\
\operatorname{Norm}(\mathrm{s})=1-1 / \mathrm{s}\end{array}$ & $\begin{array}{l}\text { For given acuity } \mathrm{v} \text { and size of image or } \\
\text { text } \mathrm{y} \text {, new size } \mathrm{Y}=\text { size } / \mathrm{v} \\
\text { For given contrast sensitivity s, original } \\
\text { contrast } \mathrm{c} 1 \text {, the required new contrast is } \\
1 / \mathrm{s} \text {. Choose appropriate contrast level } \mathrm{c} 2 \\
\text { closest to } 1 / \mathrm{s} \text {. } \\
\text { New norm }(\mathrm{v})=\mathrm{y}^{*} \mathrm{v} / \text { size; } \\
\text { New norm }(\mathrm{c})=\mathrm{c} 2 \text {; }\end{array}$ \\
\hline $\begin{array}{l}\text { Diabetic Retinopathy and } \\
\text { Macular Degeneration }\end{array}$ & $\begin{array}{l}\text { Macula, retina and } \\
\text { fovea resulting in } \\
\text { loss of } \quad \text { detail, } \\
\text { distortion } \\
\text { reduced and } \\
\text { perception }\end{array}$ & $\begin{array}{l}\text { Visual acuity (v), } \\
\text { contrast sensitivity } \\
(\mathrm{s}) \text {, color(c) } \\
\text { Normalization for } \mathrm{v} \\
\text { and c same as above } \\
\text { Norm }(\mathrm{c})= \\
(\mathrm{X}+\mathrm{Y}+\mathrm{Z}) / 3\end{array}$ & $\begin{array}{l}\text { Transcoding for factors } \mathrm{v} \text { and s same as } \\
\text { above } \\
\text { For given responses } \mathrm{X}, \mathrm{Y}, \mathrm{Z}, \mathrm{RGB} \text { color } \\
\text { values calculated and closest color level } \\
\text { chosen. } \\
\text { New norm(c) = Total sensitivity of } \\
\text { Selected colors/no of colors }\end{array}$ \\
\hline $\begin{array}{l}\text { Glaucoma, } \\
\text { Pigmentosa }\end{array}$ & $\begin{array}{l}\text { Retina, optic nerve, } \\
\text { resulting in poor } \\
\text { peripheral vision and } \\
\text { brightness perception }\end{array}$ & $\begin{array}{l}\text { Field of View } \\
(\theta), \text { brightness }(b) \\
\operatorname{Norm}(\theta)=\theta / 60 \\
\operatorname{Norm}(b)=1-1 / b\end{array}$ & $\begin{array}{l}\text { For field } \theta \text { and size of image in } \mathrm{x} \\
\text { dimension, new size }=\frac{\theta^{*} \mathrm{x}}{60} \text {; } \\
\text { Norm }(\theta)=\left(\left(\mathrm{x}^{*} \theta\right) / \text { new size }\right) / 60 \\
\text { For brightness b the selected intensity } \\
\text { level is } \mathrm{e}^{\mathrm{b}} \text {; For selected intensity I new } \\
\text { norm }(\mathrm{b})=1-(1 / \log )\end{array}$ \\
\hline Color Blindness & $\begin{array}{l}\text { Cones, can affect red, } \\
\text { green or blue cones }\end{array}$ & $\begin{array}{l}\text { Color Perception }(\mathrm{c}) \\
\text { Norm }(\mathrm{c})= \\
(\mathrm{X}+\mathrm{Y}+\mathrm{Z}) / 3\end{array}$ & $\begin{array}{l}\text { For given responses } \mathrm{X}, \mathrm{Y}, \mathrm{Z}, \mathrm{RGB} \text { color } \\
\text { values calculated and closest color level } \\
\text { chosen. } \\
\text { New norm(c) = Total Sensitivity of } \\
\text { selected colors/no of colors }\end{array}$ \\
\hline
\end{tabular}

$$
\begin{aligned}
& X=0.6067 R+0.1736 G+0.2001 B \\
& \mathrm{Y}=0.2988 \mathrm{R}+0.5868 \mathrm{G}+0.1143 \mathrm{~B} \\
& \mathrm{Z}=0.0000 \mathrm{R}+0.0661 \mathrm{G}+1.1149 \mathrm{~B}
\end{aligned}
$$

Visual Field $(\theta)$ : the visual field is the total area where objects can be seen in the peripheral vision while the eye is focused on a central point. Each eye has a horizontal field of view of $150 \mathrm{deg}$ ( $60 \mathrm{deg}$ towards the nose, $90 \mathrm{deg}$ to the side) and a vertical field of view of $120 \mathrm{deg}$. A visual field of $20 \mathrm{deg}$ indicates blindness. Visual field affects the visual angle of the user, which influences the amount of information received by the eye.

For effective adaptation we characterize the factors that affect different vision impairments like glaucoma, refractive errors etc, and apply the appropriate transcoding policy to the distant learning application as appropriate. Table 1 summarizes the transcoding policies required for different impairments.

\subsection{Visibility Based Adaptation Policies}

As seen in Section 2, the visibility of the given information is dependent on a number of factors. The effect of different impairments with respect to the above factors is given in $[9,4,8,5,7,6]$. For effectively characterizing the visibility of the given information we introduce a term called visual accessibility VA. The visual accessibility is defined as a function of the different factors as described in the previous section.

$$
\mathbf{V A}=\mathrm{f}(V, S, B, C, \theta)=\mathrm{N}(V)+\mathrm{N}(S)+\mathrm{N}(B)+\mathrm{N}(C)+\mathrm{N}(\theta)
$$

where $\mathrm{N}(\mathrm{i})$ is a normalized value for each of these factors, and $0<=\mathrm{N}(\mathrm{i})<=1$. For simplicity, we first normalize each of these factors to fit into a uniform scale and sum the normalized values to obtain a measure of visual accessibility. Our goal is to maximize the visual accessibility for users with varying degrees and types of vision impairments. 
The normalization process is specific to the parameter; we reduce each factor to a numerical value between 0 and 1 where 0 represents a high degree of impairment and 1 represents the value of that factor for a normal unimpaired user. Modeling brightness and contrast sensitivity, is very complex and involves detailed experiments, which we will be pursuing as part of our future goals. Hence the visual accessibility of the distance-learning environment is primarily dependent on the other three factors, i.e., visual acuity, visual field and color perception. We propose adaptation policies that will improve visual accessibility. The enforcement of the adaptations can be achieved by a combination of serverside transcoding techniques and client-side user interface adaptations. In either case, the content source is the best point to make transcoding decisions, since the adaptations are content specific.

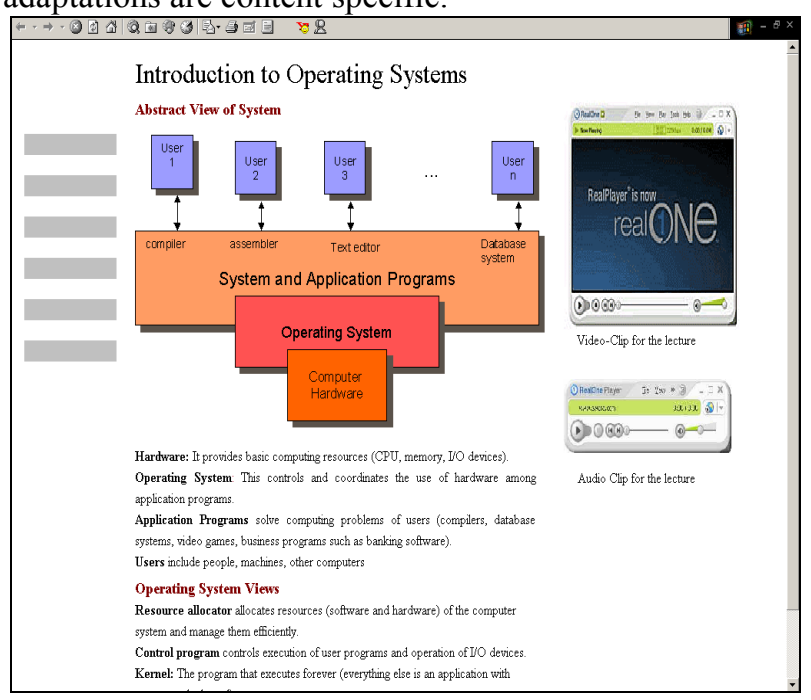

Figure 1: Sample Distance Learning Portal

To illustrate the adaptation policies we depict a distancelearning portal (DLP) with 3 different regions as illustrated in Figure 1. Such a distance-learning portal is being developed under Project SUGA at the University of California, Irvine ${ }^{1}$. The side panel (region 1) contains the outline of the website, the main course content is in the center (region 2), and the panel consisting of the video and audio clips is on the right (region 3). In principle we can extend this to any number of regions. The transcoding policy depends on the priority of the different regions as assigned by the designer. However here we assume the different regions have equal priorities. The dimensions of the original DLP for the region Ri (where $\mathrm{i}$ $=1$ to number of regions) are $X_{R i}$ and $Y_{R i}$, with viewable screen dimensions being Sx and Sy. The color of the image and video is given by the R, G, B values. The font size for all regions is given by tf.

\footnotetext{
${ }^{1}$ http://www.ics.uci.edu/ dsm/suga/
}

Consider an arbitrary user with visual acuity v and visual field $f$ and and color perception values for red, green and blue being $\mathrm{X}, \mathrm{Y}$, and $\mathrm{Z}$ respectively. We only consider the horizontal field of view towards the nose accounting for central vision ${ }^{2}$. Furthermore, the color perception values are 1 or 0 to only account for total presence or absence of sensitivity to a certain color. We assume that the user provides his/her visual profile during a separate profiling session. Specific details of the profiling process are beyond scope of this paper.

Figure 2 illustrates the adaptation algorithms for 2 specific classes of vision impairments. Case 1 is the transcoding policy when visual acuity is low (as is the case with refractive errors), and Case 2 is the policy for low visual field. The color perception is accounted for in both these cases by filtering out the color that is inaccessible. The layout of the portal remains is not changed to deal with color blindness.

To prevent the size of the regions becoming too small we use content-specific threshold factors $(\lambda)$ that determine the minimum size of a region. For a primarily text-based region, $\lambda$ is given by a minimum specified number of characters per line. If the region's content consists of images and video, $\lambda$ specifies the minimum viewable screen or image size. $\lambda_{\mathrm{Ri}}$ denotes the threshold for the given region while $\lambda_{\mathrm{S}}$ represents the threshold size of the viewable screen.

There are also intricate dependencies between the different factors. To simplify the transcoding process, we only model those dependencies that have a significant impact on visual perception. Furthermore, the transcoding decisions may conflict in the case when multiple visual factors are impacted. For instance, a low visual acuity requires enlargement of image/text while a low field of vision will trigger a size reduction. The transcoding system must contain rules to handle such conflicts; for instance, in the above case, a bounded enlargement factor to suit the feasible field of view must be calculated. We are currently working on techniques that consider the existence of such conflicting factors.

\section{Results of Adaptive Transcoding}

In this section we can see how the transcoding policies defined in the previous section help modify the existing environment to suit the different vision impairments. Let us consider an environment with three regions as specified in the previous section. Using the transcoding policies we modify the DLP as appropriate to the user.

\footnotetext{
${ }^{2}$ We do not account for peripheral field of view, as it is not critical for the current application i.e. to access multimedia information over the network.
} 


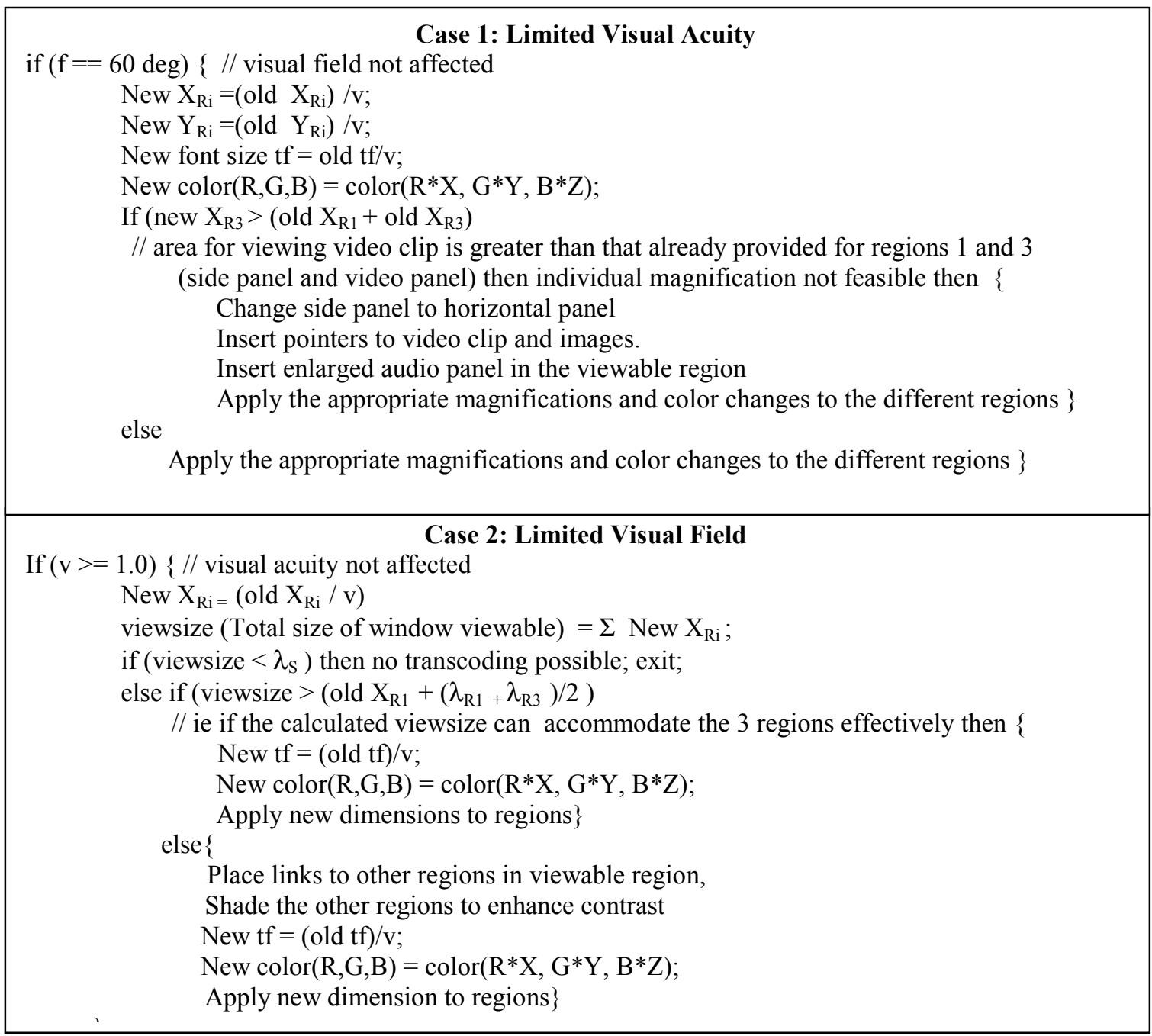

Figure 2: Visibility Based Adaptation Algorithms

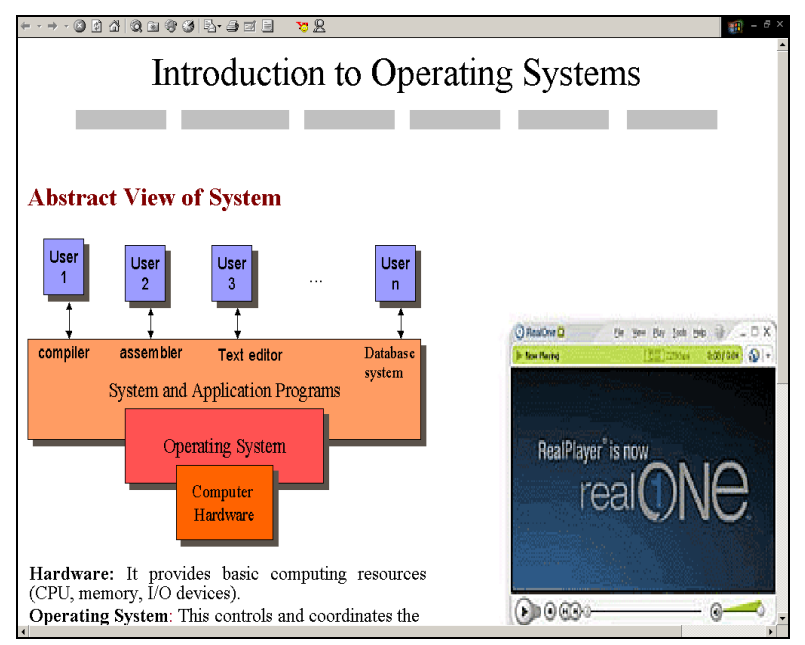

Figure 4: Screenshot for a user with visual acuity 20/30

For a user with refractive errors like myopia, hyperopia and presbyopia, the visual acuity is affected. To increase the accessibility, magnification is necessary. If the magnification of one region results in reduction of the other we shift this region as specified in the policy.Figure 4 shows how the page is adapted to a user with a corrected visual accessibility of 20/30. Notice the side panel (region 1) is now remodeled as a horizontal panel. Appropriate magnification of the video clip, image and font are also applied.

For a very low visual acuity, the magnification required is often more than double the original size. In such cases, if the original image or video cannot fit into the given region, a thumbnail representation is included, which can be magnified in a new window (see Figure 5). This is done so that the main content of the course is not affected and remains accessible to the user. Notice the thumbnails and the enlarged audio clip are now part of main viewable region. Furthermore, note the magnified hover buttons and font size. 

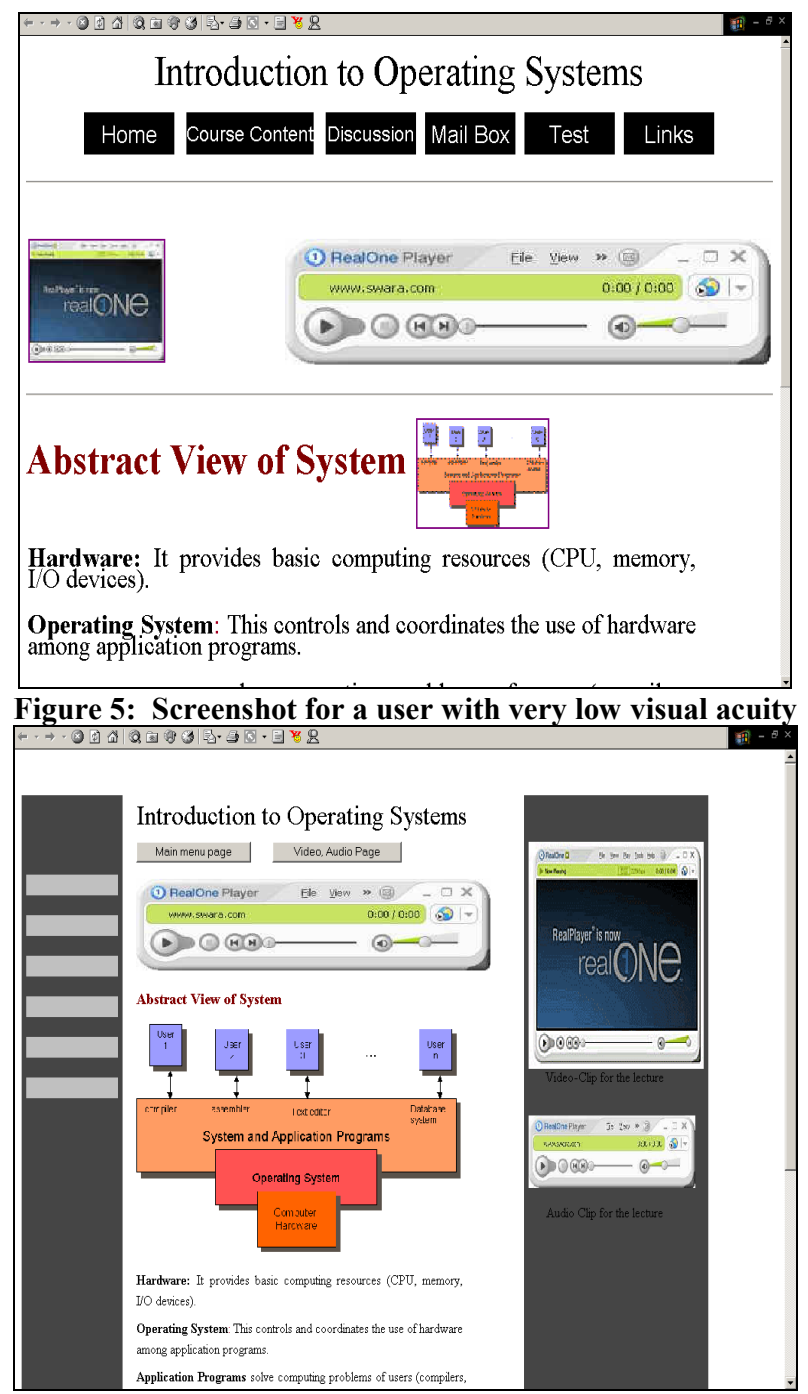

Figure 6: Screenshot for a user with low visual field

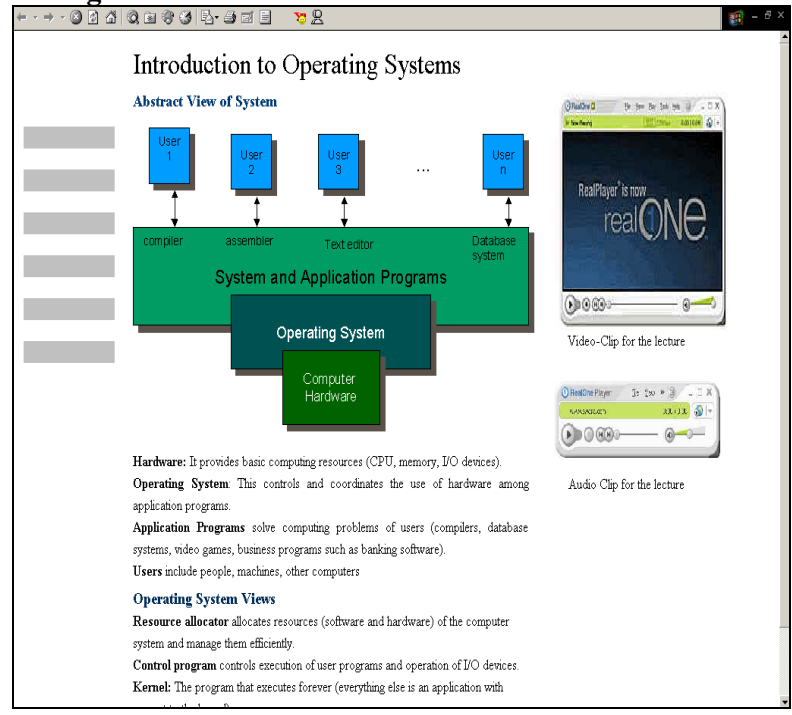

Figure 7: Screenshot for users with red color blindness
For visual field errors, we reduce the overall width of the region. For a small degree of impairment, transcoding involves horizontal shrinking of regions with no loss of content. For higher degrees of visual field impairment, we designate the central region as the viewable screen and accommodate critical content within the same. As can be seen from Figure 6, the side panes are shaded to increase viewability, and the links are introduced to point to the surrounding regions (side and video panels). The audio panel is brought to the main region. For color blindness, the appropriate color that is not accessible is filtered out. Figure 7 shows how the image colors are changed to increase the accessibility to a user who cannot differentiate reds. The filtering out of the red color results in other viewable shades as shown.

\section{Conclusions}

The adaptation of the distance-learning portal to suit different users with varied degrees of vision impairments is essential to enhance to usability of a distance learning application. In this paper, we used models of the human visual system to determine transcoding parameters that can significantly improve accessibility of distance learning information to visually impaired users. The visibility based adaptation algorithm proposed is being extended further to accommodate contrast sensitivity and brightness perception factors. Future work also addresses the extension of the distancelearning portal for cross-disability access - specifically, to users with cognitive and motor impairments.

\section{References}

[1]ContrastSensitivity.net, http://www.contrastsensitivity.net, 2002

[2] Fraser and Gutwin, “ A Framework of Assistive Pointers for Low Vision Users" ACM Assets 2000

[3]Image Processing, http://www.mathworks.com/access , 2002

[4] Lighthouse International, http://www.lighthouse.org, 2002

[5] MEDLINEplus: Refractive Errors, http://www.nlm.nih.gov/medlineplus/refractiveerrors.html, 2002

[6] Retina International, http://www.retina-international.org/, 2002

[7] Royal National Institute for the Blind, UK, http://www.rnib.org, 2002

[8] D. S.Ray, E.J.Ray, "Adaptive Technologies for the Visually Impaired: The Role of Technical Communicators", Journal of the Society for Technical communication", 1998

[9] http://www.sapdesignguild.org/resources

[10] Jim Thatcher, "Screen Reader/2 Access to OS/2 and the Graphical User Interface”, ASSETS'94

[11] Vision Channel, http://www.visionchannel.net, 2002

[12] Visicheck , http://www.vischeck.com, 2002

[13] American Optometric Association, http://www.aoanet.org, 2002

[14] Gerhard Weber, "Programming for Usability in Nonvisual User Interfaces”, ASSETS'98

[15] Frankie James, "Lessons from Developing Audio HTML Interfaces", ASSETS'98 